\title{
Ancestry-specific associations identified in genome-wide combined-phenotype study of red blood cell traits emphasize benefits of diversity in genomics
}

Chani J. Hodonsky ${ }^{1,2^{*}}$ (D), Antoine R. Baldassari ${ }^{1}$, Stephanie A. Bien ${ }^{3}$, Laura M. Raffield ${ }^{4}$, Heather M. Highland ${ }^{1}$, Colleen M. Sitlani ${ }^{5}$, Genevieve L. Wojcik ${ }^{6}$, Ran Tao ${ }^{7}$, Marielisa Graff', Weihong Tang ${ }^{8}$, Bharat Thyagarajan', Steve Buyske ${ }^{9}$, Myriam Fornage ${ }^{10}$, Lucia A. Hindorff' ${ }^{11}$, Yun Li ${ }^{1}$, Danyu Lin ${ }^{1}$, Alex P. Reiner ${ }^{3,12}$, Kari E. North ${ }^{1,4}$, Ruth J. F. Loos ${ }^{13}$, Charles Kooperberg ${ }^{12}$ and Christy L. Avery ${ }^{1}$

\begin{abstract}
Background: Quantitative red blood cell (RBC) traits are highly polygenic clinically relevant traits, with approximately 500 reported GWAS loci. The majority of RBC trait GWAS have been performed in European- or East Asian-ancestry populations, despite evidence that rare or ancestry-specific variation contributes substantially to RBC trait heritability. Recently developed combined-phenotype methods which leverage genetic trait correlation to improve statistical power have not yet been applied to these traits. Here we leveraged correlation of seven quantitative RBC traits in performing a combined-phenotype analysis in a multi-ethnic study population.

Results: We used the adaptive sum of powered scores (aSPU) test to assess combined-phenotype associations between $\sim 21$ million SNPs and seven RBC traits in a multi-ethnic population (maximum $n=67,885$ participants; 24\% African American, 30\% Hispanic/Latino, and 43\% European American; 76\% female). Thirty-nine loci in our multiethnic population contained at least one significant association signal $(p<5 \mathrm{E}-9)$, with lead SNPs at nine loci significantly associated with three or more RBC traits. A majority of the lead SNPs were common (MAF $>5 \%$ ) across all ancestral populations. Nineteen additional independent association signals were identified at seven known loci (HFE, KIT, HBS1LMYYB, CITED2/FILNC1, ABO, HBA1/2, and PLIN4/5). For example, the HBA1/2 locus contained 14 conditionally independent association signals, 11 of which were previously unreported and are specific to African and Amerindian ancestries. One variant in this region was common in all ancestries, but exhibited a narrower LD block in African Americans than European Americans or Hispanics/Latinos. GTEx eQTL analysis of all independent lead SNPs yielded 31 significant associations in relevant tissues, over half of which were not at the gene immediately proximal to the lead SNP.

(Continued on next page)
\end{abstract}

\footnotetext{
* Correspondence: ch2um@virginia.edu

'University of North Carolina Gillings School of Public Health, 135 Dauer Dr, Chapel Hill, NC 27599, USA

¿University of Virginia Center for Public Health Genomics, 1355 Lee St, Charlottesville, VA 22908, USA

Full list of author information is available at the end of the article
}

(c) The Author(s). 2020 Open Access This article is licensed under a Creative Commons Attribution 4.0 International License, which permits use, sharing, adaptation, distribution and reproduction in any medium or format, as long as you give appropriate credit to the original author(s) and the source, provide a link to the Creative Commons licence, and indicate if changes were made. The images or other third party material in this article are included in the article's Creative Commons licence, unless indicated otherwise in a credit line to the material. If material is not included in the article's Creative Commons licence and your intended use is not permitted by statutory regulation or exceeds the permitted use, you will need to obtain permission directly from the copyright holder. To view a copy of this licence, visit http://creativecommons.org/licenses/by/4.0/ The Creative Commons Public Domain Dedication waiver (http://creativecommons.org/publicdomain/zero/1.0/) applies to the data made available in this article, unless otherwise stated in a credit line to the data. 


\begin{abstract}
(Continued from previous page)
Conclusion: This work identified seven loci containing multiple independent association signals for RBC traits using a combined-phenotype approach, which may improve discovery in genetically correlated traits. Highly complex genetic architecture at the HBA1/2 locus was only revealed by the inclusion of African Americans and Hispanics/ Latinos, underscoring the continued importance of expanding large GWAS to include ancestrally diverse populations.
\end{abstract}

Keywords: Blood cell traits, Combined-phenotype analysis, Pleiotropy, Diversity, Multi-ethnic, GWAS

\section{Background}

In the average adult, 200 billion red blood cells (RBCs) are generated daily from hematopoietic stem cells in the bone marrow. The most commonly assessed traits for mature RBCs are hematocrit (HCT), hemoglobin concentration (HGB), mean corpuscular hemoglobin (MCH), $\mathrm{MCH}$ concentration $(\mathrm{MCHC})$, mean corpuscular volume (MCV), RBC count (RBCC), and red cell distribution width (RDW); together, these traits are used to characterize RBC development and function, diagnose anemic disorders, and identify risk factors for complex chronic diseases [1-6]. RBC traits also are moderately to highly heritable, making these complex quantitative traits excellent candidates for genomic interrogation [7-9]. Improved characterization of RBC molecular pathways has benefitted both disease diagnosis and pharmaceutical development, as has been demonstrated by recent successes in a BCL11A-silencing gene therapy clinical trial for individuals with sickle cell disease (SCD) $[10,11]$.

Genetic association studies have reported over 500 independent loci for RBC traits [12-31]. However, several research gaps remain which may be addressed via recently developed methods and broadly representative study populations. First, previously published RBC trait genome-wide association study (GWAS) populations have mostly been ancestrally homogeneous [31-39]. Utilization of diverse study populations can improve identification of rare or ancestry-specific variants located in biological pathways that affect phenotypes in global populations and, when summary data are made publicly available, enable construction of broadly applicable polygenic risk scores [40]. Relatedly, gaps between estimated heritability and the proportion of variance explained by GWAS findings suggest that additional associations remain to be identified, including rare variants and independent secondary associations at known loci that are both more likely to be ancestrally specific [12, 41, 42]. Finally, RBC traits exhibit modest to high correlation, and several dozen loci have been reported for two or more RBC traits, although few studies have leveraged this shared genetic architecture to increase statistical power to map novel RBC trait loci [12, 20, 26, 43-45].

In this work, we examined the individual and shared genetic architecture of seven $\mathrm{RBC}$ traits in participants of the ancestrally diverse Population Architecture using Genomics and Epidemiology (PAGE) study [46]. Our findings reinforce the necessity of incorporating multi-ethnic study populations in genomics in order to accurately characterize RBC trait loci and encourage equitable application of the results to translational work [39]. The complexity of association signals at loci previously characterized in European- and East Asianancestry populations also demonstrates improved power to perform conditional analysis using a combined-phenotype model [47].

\section{Results}

The number of participants with both phenotype and genotype data ranged from 33,549 (RDW) to 67,885 (HCT, see Methods, Tables S2 \& S3). Seventy-eight percent of participants were female and participants were on average 57 years old at time of blood collection (Table S4). Self-reported race/ethnicity in the total study population was approximately $20 \%$ African American, 30\% Hispanic/Latino, and 40\% European American (Table S3).

\section{Combined-phenotype analyses}

Approximately $21 \mathrm{M}$ SNPs met our inclusion criteria and were evaluated in our primary analysis, a combinedphenotype multi-ethnic meta-analysis of seven RBC traits. SNP associations with the combined phenotype multiethnic meta-analysis exceeded genome-wide significance at 39 loci $(p<5 \mathrm{E}-09$, Figures S1, S2), all of which were identified previously. Lead SNPs at nine loci (KIT, HFE, $H B S 1 L / M Y B, I K Z F 1, T F R 2, H B B, H B A 1 / 2, G C D H$, and TMPRSS6) were associated with three or more RBC traits at genome-wide significance (Tables 1, S5A). HCT, HGB, and $\mathrm{MCHC}$ exhibited genome-wide-significant associations at the fewest loci (eleven, ten, and six, respectively), whereas $\mathrm{MCH}$ and $\mathrm{MCV}$ had the most (twenty and twenty-one, respectively, Fig. 1a, Table S5A). Estimated partial correlations by RBC trait pair ranged from HCTMCHC (partial correlation $\rho=-0.02)$ to HCT-HGB $(\rho=$ 0.94, Fig. 1b). Consistent with other GWAS of quantitative complex traits, effect size was inversely correlated to allele frequency across all phenotypes (Fig. 1c).

Trait-specific directions of effect were largely consistent with pairwise correlations. Among 58 independent 


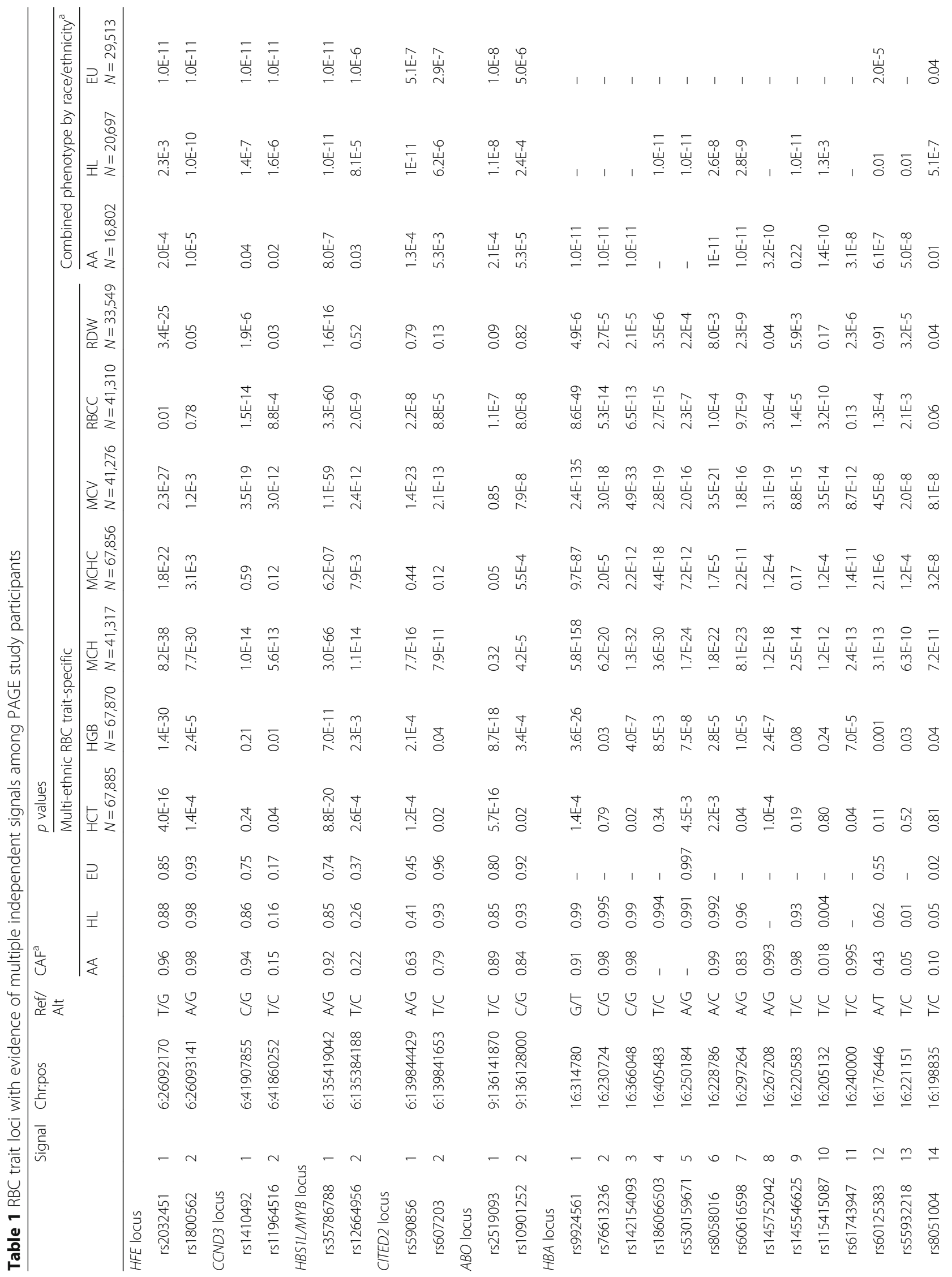




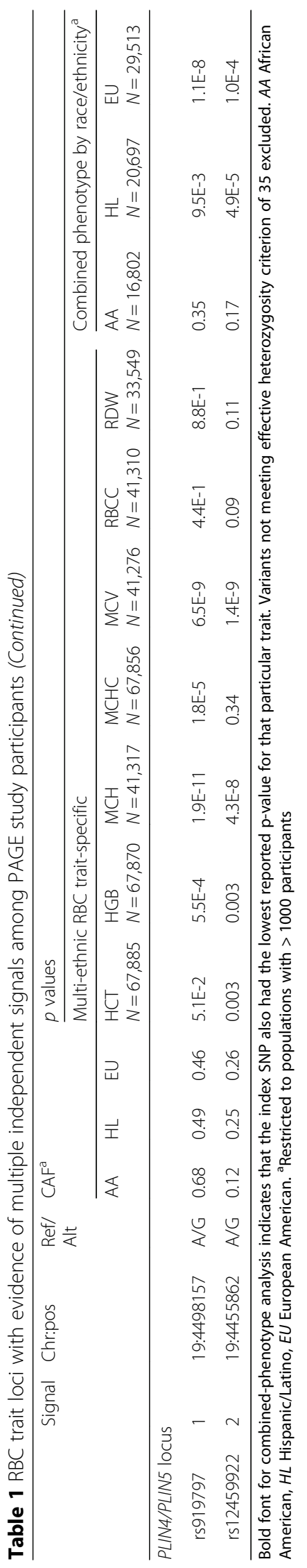




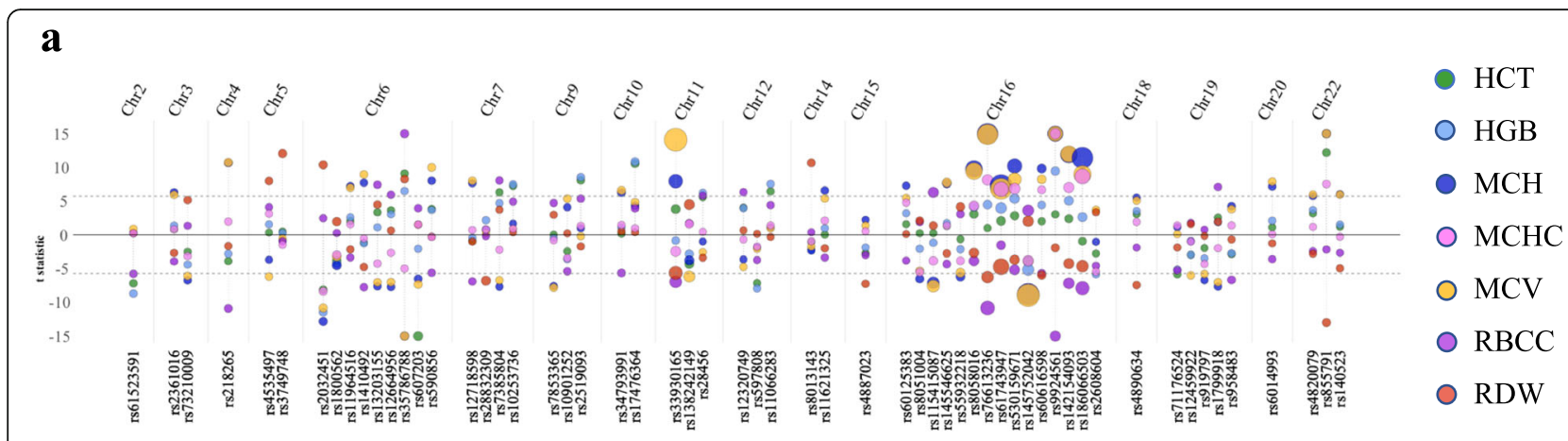

b

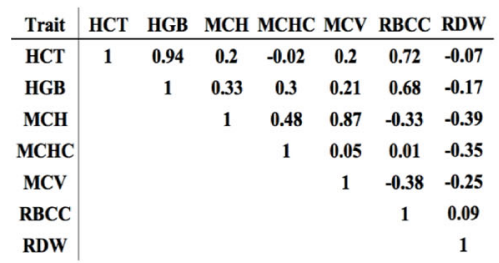

c

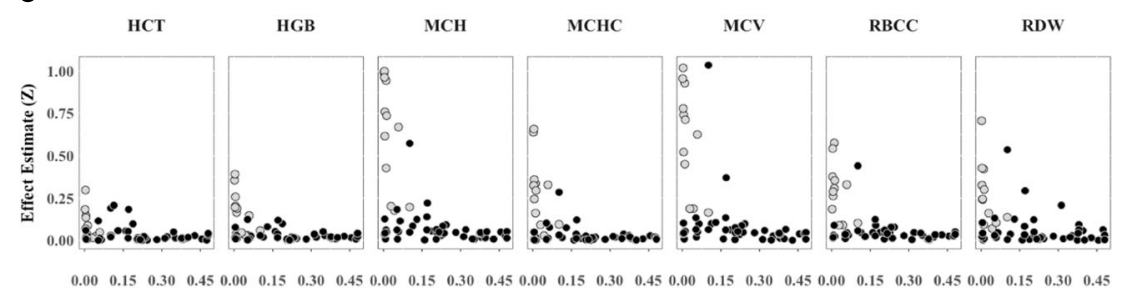

Coded Allele Frequency

Fig. 1 Identification and characterization of 58 independent lead variants in 39 loci in a multi-ethnic study population. a Lead and conditionally independent SNPs from combined-phenotype analysis of total study population show shared genetic architecture directionally consistent with correlation structure. Colored circles to the right of figure correspond to trait-specific associations. X-axis: rsid (bottom) sorted by chromosome (top) and position; $\mathrm{y}$-axis: significance of association and direction of effect, represented by $\mathrm{t}$-value (scaled to a maximum of $\mathrm{t}=|15|$ ). Size of circles is exponentially proportional to effect size standardized to trait means $\left(3^{\mathrm{Z}}\right)$ to demonstrate differences in average effect size at lead SNPs by trait. Dashed gray lines correspond to genome-wide-significance threshold of $a=5 \mathrm{E}-09$. b RBC trait pair partial correlations among MEGAgenotyped participants adjusted for linear regression model covariates $(n=29,090$ for HCT, HGB, and MCHC measurements; $n=22,330$ for MCH, $\mathrm{MCV}$, and RBCC; $n=19,573$ for RDW). c. Low-frequency and rare alleles exhibit larger magnitude of effect across RBC traits in the total multiethnic study population. X-axis: minor allele frequency; $y$-axis: effect size standardized to trait mean (|Z|). Filled circles represent variants present in all ancestry sub-populations; open circles are monomorphic in one or more ancestries

association signals identified via conditional analysis, $64 \%$ ( $n=37$ ) exceeded genome-wide significance for the combined-phenotype lead SNP in two or more traits. When comparing genome-wide significant associations for two traits exhibiting a pairwise correlation $>|0.2|$ among these loci, in 93\% of instances (119 of 128) the direction of effect matched the direction of trait correlation (Fig. 1a, b, Tables S5A, S6). Eight of nine trait-pair associations with directions of effect opposite of expectation were instances in which $\mathrm{MCH}$ or MCV drove the lead SNP association, and HCT or HGB had a different lead SNP in high LD with the combined-phenotype lead SNP $\left(\mathrm{r}^{2}>0.8\right.$ in the combined MEGA-genotyped study population). Only one of nine associations was in a trait pair exhibiting moderate correlation: HGB and RBCC $(\rho=0.68)$ exhibiting opposite directions of effect for rs9924561, the lead SNP in the $H B A 1 / 2$ region on chromosome 16.

\section{Evidence of independent associations at established loci}

We identified 20 independent association signals at seven loci (HFE, CCND3, HBS1L/MYB, CITED2, ABO, $H B A 1 / 2$, and PLIN4/5, Table 1, Fig. 1a). The majority of lead SNPs were common to all ancestries (MAF > 0.01); evidence of association was most significant in European Americans at HFE and HBS1L/MYB loci, whereas Hispanics/Latinos had the most significant association at both CITED2 lead SNPs. In two instances, known causal variants accounted for the entire association signal after conditioning. At the HFE locus, both rs1800562 (HFE p.C282Y) and rs1799945 (HFE p.H63D, $r^{2} \sim 0.99$ with lead SNP rs2032451) are known coding hemochromatosis variants and accounted for all significant associations within $+/-3 \mathrm{Mb}$ of the lead SNP [48]. Similarly, rs2519093 and rs10901252 are in moderate to high LD with variants that affect RBC traits but also determine an individual's ABO blood type, and adjusting for these two variants accounted for the entire association at this locus.

Of note, the $H B A 1 / 2$ locus demonstrated ancestry specificity (i.e., the lead SNP was monomorphic in one or more ancestries) at 11 of 14 conditionally independent SNPs (Fig. 2a, Tables S5B-D). With the exception of rs60125383 (frequency of the A allele: 0.43 in African Americans, 0.55 in European Americans, 0.62 in Hispanics/Latinos), located in a nonsense-mediated-decay transcript for NPRL3, no lead SNP at this locus was common to all ancestries. The LD block for rs60125383 


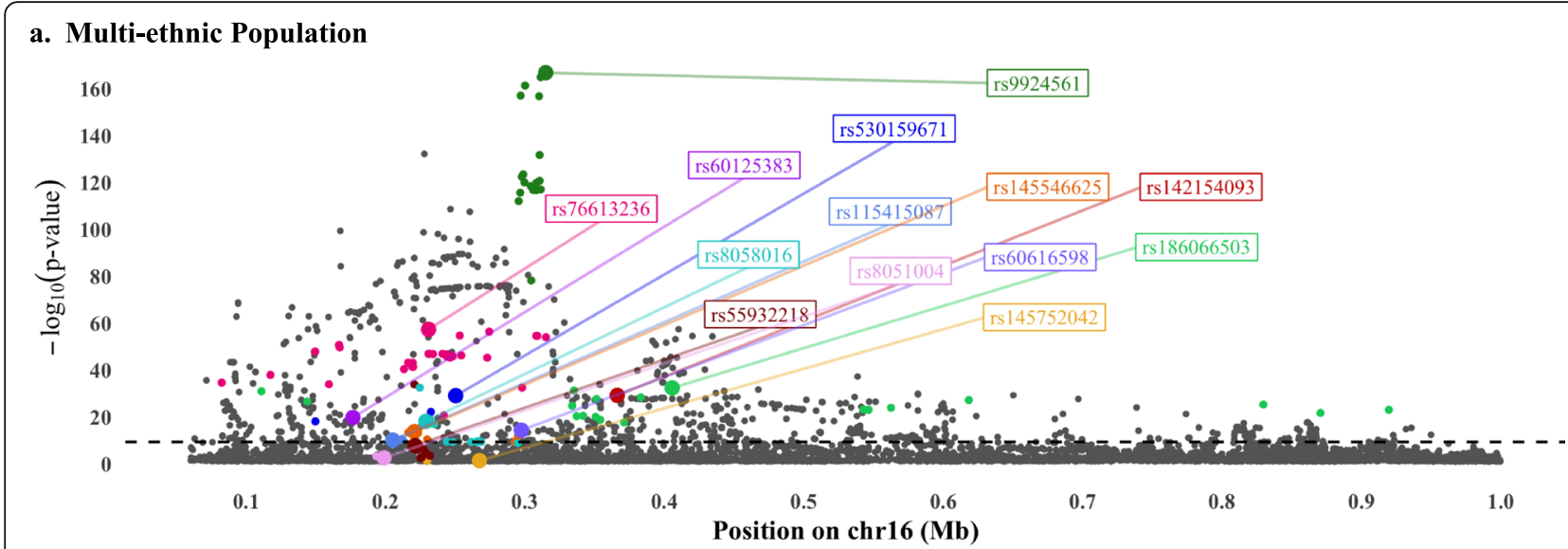

b. $\mathbf{r s 6 0 1 2 5 3 8 3}$ in African Americans

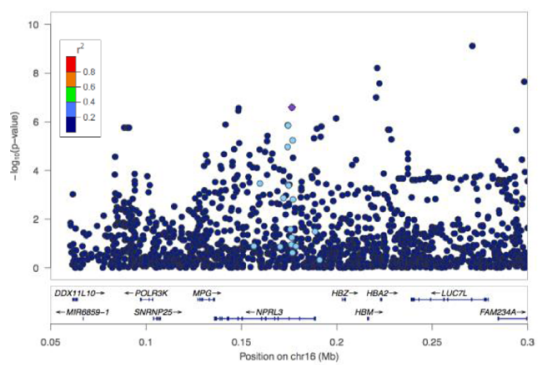

c. $\mathbf{r s 6 0 1 2 5 3 8 3}$ in Hispanics/Latinos

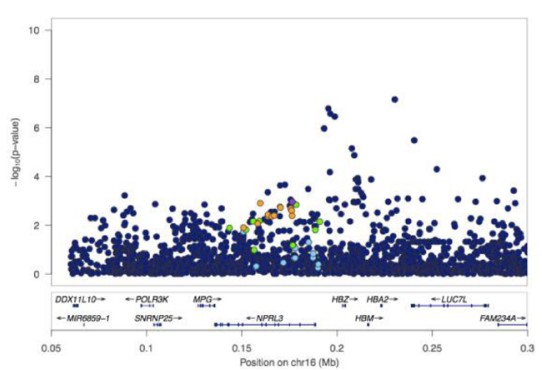

d. rs60125383 in European Americans

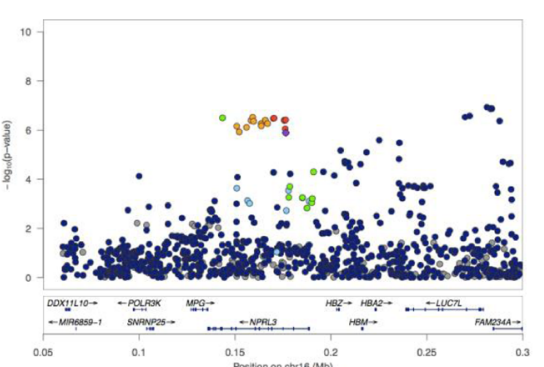

Fig. 2 Multiple independent associations with MCH demonstrate complex genetic architecture at HBA1/HBA2 locus. All plots: each point represents one SNP; $x$-axis: increasing position on chromosome 16 left to right; $y$-axis: - $\log _{10}(p$-value) of the association with $\mathrm{MCH}$. a Regional association plot of 14 independent associations in unadjusted analysis of multi-ethnic study population $(n=41,317)$. Large circles represent conditionally independent lead SNPs, labeled by rsid (order of conditioning is shown in Table 1); small colored SNPs represent variants in high LD $\left(r^{2}>0.8\right.$ in LD in pooled MEGA subpopulation) with the lead SNP of the corresponding color. $\mathbf{b}$ - $\mathbf{d}$ Locus-Zoom regional association plots of MCH association with rs60125383 (11th round of conditioning, purple diamond) in African Americans on an African American LD background (b $n=$ 8703), Hispanics/Latinos on a Hispanic/Latino LD background (c, $n=17,380)$, and European Americans on a European LD background (d $n=$ 14,707). SNP correlation with the lead SNP $\left(r^{2}\right)$ is colored according to the legend in (b). Annotated Refseg genes proximal to the lead SNP are shown by position above the $X$ axis

contained fewer variants in African Americans (Fig. 2b, no SNPs $r^{2}>0.4$ ) compared to Hispanics/Latinos (Fig. 2c, 10 SNPs $r^{2}>0.6$ ) and European Americans (Fig. 2d, 13 SNPs $\left.\mathrm{r}^{2}>0.6\right)$.

\section{Sensitivity analyses}

Trait-specific sensitivity analyses identified two previouslyunreported variants exceeded genome-wide significance for a single $\mathrm{RBC}$ trait in the univariate analyses, yet did not meet genome-wide significance in the combined phenotype. Rs6573766 was specific to RBCC $(p=1.1 \mathrm{E}-9)$ and is common to all ancestries but was poorly captured by earlier genotyping arrays and is not represented in 1000 genomes phase 3 data (Figure S3, Table S7). Rs145548796 was significant for MCV $(p=4.6 \mathrm{E}-9)$ and is rare $(<1 \%)$ in all populations, only meeting the inclusion criteria in the MEGA pooled sample and one study sub-population (Figure S4, Table S7). Ancestry-specific sensitivity analyses did not uncover any significant association signals that did not achieve genome-wide significance in the overall study population.
When adjusting for esv3637548 deletion dosage in the MEGA-genotyped subgroup, we observed evidence of both attenuation and strengthening of effect at otherwise conditionally independent lead SNPs at the $H B A 1 / 2$ locus (Table S8). Specifically, eight lead SNPs lost more than two orders of magnitude $p$-value after conditioning on esv3637548; one increased in significance; and five remained unchanged. Among the lead SNPs in this chromosomal region which remained significant was rs145546625, which was previously reported as significant for MCV independent of esv3637548 in a GWAS of HCHS/SOL participants using a different genotyping array [28]. All other PAGE lead SNPs in the $H B A 1 / 2$ region either did not pass QC or imputation criteria for the custom array used in that study, or had $p>1 \mathrm{E}-07$ in the primary analysis.

\section{Generalization of previously reported associations}

Generalization of previously identified association signals varied for trait-specific loci $(p<1.07 \mathrm{E}-4$, Tables S9-S11), 
ranging from 50 of 143 (35\%) for $\mathrm{MCHC}$ to 93 of 121 (77\%) for HGB. Ancestry-specific generalization varied by trait, with the highest proportion of generalization occurring in the European-ancestry sub-population and the lowest occurring in African Americans, which may be due to power differences to detect associations by ancestry.

\section{eQTL function of index SNPs}

To assess the potential regulatory roles of lead SNPs, we evaluated cis-eQTL $(<500 \mathrm{~kb})$ associations for all lead SNPs in GTEx as available [49]. Thirty-three of 51 SNPs were low-frequency or common (MAF $>1 \%$ ) in the European-ancestry GTEx population and had available information in whole blood, liver, spleen, and/or thyroid tissues. Fourteen SNPs exhibited significant associations in RBC-relevant tissues; seven SNPs were eQTLs for multiple genes (Table S12). Although approximately 40 genes were within $500 \mathrm{~kb}$ of each of the chromosome 16 lead SNPs, none of the lead SNPs in this region exceeded a MAF $>1 \%$ in the GTEx study population and hence could not be evaluated for cis-eQTLs.

\section{Discussion}

$\mathrm{RBC}$ traits are complex quantitative phenotypes that have been broadly examined in GWAS of European- and East Asian-ancestry study populations. Here, we examine the benefits of identifying and characterizing RBC trait associations in the ancestrally diverse PAGE study population using a combined-phenotype approach. Although the combined-phenotype method we employed did not enable identification of novel loci, ancestral diversity improved characterization of loci containing both ancestry-specific and common variants. The continued underrepresentation of diverse populations in GWAS despite the growing clinical and public health significance of GWAS-enabled tools that are ancestry-specific underscores the continued importance of expanding existing RBC trait GWAS of predominantly European and East Asian populations to global populations [50-53].

With regard to regions exhibiting multiple independent significant associations, our results demonstrate allelic heterogeneity at known RBC trait loci, the characterization of which was enabled by an inclusive study design. Of particular note was our identification of eleven variants specific to African and/or Amerindian ancestries within the first megabase of chromosome 16 . The chromosome 16 region includes hemoglobin genes $H B A 1, H B A 2, H B M$, and $H B Z$ as well as fifty other protein-coding genes that should be examined for plausible roles in RBC trait biology. Decades of research have demonstrated selective pressure in this region occurring over millennia in malaria-endemic regions of the world but, as with many other complex quantitative traits, red blood cell traits-specifically with regard to the $H B A 1 / 2$ locus-have been primarily analyzed in Eurocentric study populations. Given the high polygenicity and complexity of quantitative RBC traits, our identification of over a dozen independent association signals suggests a highly-transcribed region with either complementary or redundant regulatory mechanisms that may affect multiple genes. Future work could extend our efforts by examining other populations in malaria-endemic regions, as well as previously identified and highly influential structural variants, including a previously identified $3.7 \mathrm{~kb}$ copy number variant, which we were only able to evaluate as a sensitivity analysis [28].

A combined-phenotype method was selected due to its purported ability to increase statistical power to identify novel loci with modest effects across multiple correlated traits. However, sample sizes of previous RBC trait GWAS suggest that many loci with modest effects and lead SNPs in the low to common allele frequency range in European or East Asian populations have already been identified. Power was also lacking to detect loci that might be specific to other race/ethnic groups-although African Americans and Hispanics/Latinos were wellrepresented in this study, sample sizes similar to European populations will not be proportionately representative of genetic diversity, particularly for variants that are low-frequency or difficult to impute. This observation demands an increase in representation of African Americans and Hispanics/Latinos, as narrower (on average) LD blocks in populations exhibiting ancestral admixture also improve fine-mapping for prioritizing candidate variants for functional characterization. A combinedphenotype method can also improve the interpretability of association signals when one causal SNP per association signal is assumed. For example, a direction of effect inconsistent with the phenotypic correlation of two RBC traits is feasible in some anemia states, for which $\mathrm{MCV}$ and RDW-despite being negatively correlated in healthy individuals-may vary widely depending on the underlying cause [54, 55]. The African-ancestry-specific SNP rs9924561 (previously identified for $\mathrm{MCH}, \mathrm{MCHC}$, and $\mathrm{MCV}$ ) is an example of a variant that unexpectedly showed opposite directions of effect for HGB and RBCC (pairwise correlation $=0.68$ ) in our study $[28,30,56]$. The mechanism driving very strong associations $(p<$ $1 \mathrm{E}-15$ in all traits aside from HCT) with this intronic variant remains uncharacterized, likely because it is not present in European-ancestry populations and hence could not be detected in otherwise highly powered studies [12, 31]. The identification of such candidate functional variants for multiple traits with the added context of the phenotypic correlation can provide insight for molecular experimentation examining causal biological mechanisms. 
The possibility that combined-phenotype methods could benefit the study of other correlated polygenic traits still merits further investigation, particularly with groups of traits that may overlap in genetic architecture, but have not been previously examined in concert. Over the past three decades, RBC traits have been associated with cardiovascular disease outcomes like heart failure and stroke, highlighting the potential for identifying novel pleiotropic loci [6, 57-62]. Indeed, combinedphenotype approaches that examine the shared genetic architecture underlying intermediate phenotypes and clinical events may be particularly powerful for outcomes like stroke and heart failure, given that phenotypic heterogeneity of these phenotypes has complicated locus identification and characterization.

Our evaluation of lead SNPs' effects on expression in RBC-relevant tissues faced known constraints that limited interpretation and contextualization of identified variants. Crucially, the vast majority of publicly available functional data were collected from European-ancestry individuals, precluding the use of these databases for interpreting potential effects of ancestry-specific or lowfrequency SNPs on gene expression. For example, rs8051004 is one of two less frequent variants that were detected in European-ancestry populations at the HBA1/ 2 locus $(C A F=0.02)$. However, rs8051004 was reported as "monoallelic" in spleen tissue in GTEx, despite having a 10\% allele frequency in PAGE African Americans and 12 and $11 \%$ in the $1000 \mathrm{G}$ African and East Asian superpopulations, respectively. The exclusion of populations with African, Amerindian, and Asian ancestry continues to hamper the potential benefits of these resources. Additionally, while the GTEx consortium has made extensive efforts to characterize a wide array of tissue types, bone marrow was not included [49]. RBCs enucleate in the bone marrow prior to entering circulation, with no nuclear transcription and extremely limited translation occurring in mature RBCs. Therefore, bone marrow is the only tissue for which eQTL data characterizing the effects of genetic variation on gene expression for RBCs directly.

As with other genetic association studies, we faced several limitations. First, sample sizes for RBC trait GWAS have ballooned to nearly 200,000 participants and we were restricted to a smaller study population. However, the PAGE study has recently demonstrated that modest-sized studies that are more ancestrally diverse improve detection of novel and independent signals compared to simply increasing the number of European-ancestry individuals [56]. Second, while this study did improve on previous studies in terms of representation from African and American continental ancestries, we were unable to evaluate associations in several populations, particularly South Asians, Pacific Islanders, Native Americans, and Native Hawaiians. Native
Americans and Native Hawaiians are represented in PAGE, but RBC phenotypes were not measured in contributing studies. South Asian study populations have been included in several previous RBC trait GWAS; Native Americans and Pacific Islanders remain underrepresented in GWAS of all complex traits [15, 20, 39, 63]. Third, we were unable to evaluate structural variants, which have traditionally been difficult to impute, and recalling all structural variants within significant loci was outside the scope of this work. A sensitivity analysis accounting for the effect of esv3637548 in MEGA-genotyped study participants suggests that further evaluation is required to determine whether true causal variants overlap the position of this $3.7 \mathrm{~kb}$ structural variant on other ancestral haplotypes. However, it is expected that some structural variants will be adequately represented by proxy SNPs, and future sequencing-based studies will be able to characterize these rare variants. Finally, eQTL data could not be comprehensively interpreted given the limitations of publicly available databases as described above. It is imperative that these resources focus their efforts on improving inclusivity over the next several years to keep abreast of increased representativeness in association studies.

\section{Conclusion}

In conclusion, we identified over 50 association signals within 39 loci in a combined-phenotype analysis of seven $\mathrm{RBC}$ traits. We did not observe large improvement in discovery signal detection by using the combinedphenotype methods, although further work is required to fully test the utility of these approaches. However, our work demonstrates the benefits of diverse study populations for highly polygenic traits, in spite of the fact that while global populations are increasing in genetic diversity, genetic research has become less diverse. As genomics tools become more broadly available, our results underscore the critical importance of including diverse global populations so the benefits of genomics research can be equitably applied.

\section{Methods \\ Study population}

The PAGE study comprises ancestrally-diverse study populations from United States cohorts and biobanks evaluating common complex diseases and accompanying risk factors (see online supplement for more information). This study used data from self-reported African American, Asian American, European American, Hispanic/Latino, and Native American participants from the Atherosclerosis Risk in Communities Study (ARIC); the Coronary Artery Risk Development in Young Adults Study (CARDIA); the Hispanic Community Health Study/Study of Latinos (HCHC/SOL); the Icahn Mt. Sinai School of Medicine BioME Biobank (BioME); and 
the Women's Health Initiative (WHI, described above). Our study population comprised sixteen analytic subgroups which were genotyped and imputed separately. Fifteen of the sixteen analytic subgroups were identified by study and self-reported race/ethnicity (Tables S2, S3). The sixteenth subgroup was a pooled sample of selfreported African American, Asian American, Hispanic/ Latino, Native American, and "Other" MEGA-genotyped individuals from BioMe, HCHS/SOL, and WHI. Participants were excluded if they had ever been diagnosed with HIV or leukemia, were pregnant at time of blood draw, were receiving chemotherapy at time of blood draw, or had a severe hereditary anemia (primarily sickle-cell disease, determined by genotype).

\section{$\mathrm{RBC}$ trait measurement}

$\mathrm{RBC}$ traits were measured with hemanalyzers following standardized laboratory protocols from blood draws at the earliest available visit (see online supplement) for the three primary (HCT, HGB, and RBCC) and four derived $(\mathrm{MCH}, \mathrm{MCHC}, \mathrm{MCV}$, and RDW) RBC traits (Table S1). $\mathrm{RBC}$ trait values that exceeded four standard deviations from the mean of the trait in the overall study population were excluded, mirroring protocols established by prior GWAS $[28,45]$. Pairwise correlation coefficients were calculated in the MEGA-genotyped analytic subgroup (see below) adjusting for all the covariates used in univariate regression analysis, specifically age at blood draw, sex, study site or region, and ancestral principal components.

\section{Genotyping, quality control, and imputation}

Genotyping methods have been described for each of our study sub-populations previously; all imputation of genotype data used in this study was performed by the PAGE coordinating center [64]. Briefly, genotyping arrays and quality control measures used were as follows. Affymetrix Genome-Wide Human SNP Array 6.0 for ARIC, BioMe Mt. Sinai Biobank European Americans, CARDIA, and WHI SHARe. The Illumina OmniExpress was used to genotype individuals for all remaining BioMe Mt. Sinai Biobank participants. WHI GARNET participants were genotyped on the Illumina Human Omni1-Quad v1-0 B array; WHI GECCO participants on the Illumina $610 \mathrm{~K}$ and Cytochip $370 \mathrm{~K}$ arrays; WHI HIPFX participants on the Illumina $550 \mathrm{~K}$ and $610 \mathrm{~K}$ arrays; WHI LLS participants on the Illumina HumanOmniExpressExome-8v1_A array; WHI MOPMAP participants on the Affymetrix Gene Titan, Axiom Genome-Wide Human CEU I Array Plate; and WHI WHIMS participants on the HumanOmniExpress Exome-8v1_B array. All remaining participants from BioMe, HCHS/SOL, and WHI were genotyped on the Illumina Infinium Expanded Multi-Ethnic Genotyping Array (MEGA).
With regard to quality control, studies employed either a 90\% (ARIC, MOPMAP) or 98\% (all other studies) SNP call-rate threshold. A sample call rate of 95\% was employed for ARIC and. A 98\% rate for MEGAgenotyped participants, with no sample call rate applied to remaining studies. Similarly, a 1E-06 HWE $p$-value threshold was employed for ARIC, and a 1E-04 threshold for MEGA-genotyped participants. Additional studyspecific genotype QC criteria are described in Table S2. All studies were imputed to the 1000 Genomes phase 3 reference panel by the PAGE coordinating center after study-specific quality control criteria were applied (Table S2, 56). We further excluded SNPs on a sub-studyspecific basis which had poor imputation quality $(<0.4)$ or an effective heterozygosity $<35$ (calculated as $2 \mathrm{x}$ CAF $\mathrm{x}$ (1-CAF) $\mathrm{x} \mathrm{N} \times$ imputation quality, where CAF is coded allele frequency and $\mathrm{N}$ is sample size).

\section{Statistical methods \\ Overall reporting of results}

Previously-reported SNPs for the seven RBC traits evaluated in this study were identified through review of the NHGRIEB GWAS Catalog [65] as of January 1, 2019, supplemented by a PubMed search. Multi-ethnic combined-phenotype results were presented as the primary findings, employing Bonferroni correction assuming $10 \mathrm{M}$ independent tests (i.e., genome-wide significance refers to $\left.\mathrm{P}_{\mathrm{aSpU}}<5 \mathrm{E}-9\right)$. We defined a locus using physical proximity $(+/-500 \mathrm{~kb}$ from the lead SNP), and we defined an association signal as the lead (most significant) SNP and proxy SNPs in local LD based on conditional independence within ten megabases. Discovery loci were defined as $\geq 500 \mathrm{~kb}$ from and conditionally independent of a variant previously reported to satisfy the field standard $p<5 \mathrm{E}-8$ for any of the seven RBC traits. Ancestry-specific and trait-specific analyses were performed as sensitivity analyses to improve interpretation of results. Complete summary-level results are available through dbGaP (phs000356).

\section{Univariate analysis}

Univariate associations for the seven RBC traits were estimated assuming an additive genetic model of inheritance and adjusting for linear effects of age at blood draw, sex, study site or region, and ancestral principal components [66]. The total MEGA-genotyped subgroup was analyzed using generalized estimating equations allowing correlated errors for first or second-degree relatives, and independent error distributions by selfreported ancestry group [67]. Linear regression was implemented in SUGEN for the other 15 analytic subgroups [67]. For each RBC trait, METAL software was used to perform inverse-variance-weighted meta-analysis across all sub-studies [68]. SNP effect heterogeneity was measured with the Cochran's Q test. SNP meta-analysis 
$p$-values were assessed by $\mathrm{RBC}$ trait by calculating genomic inflation factors $(\lambda)$ and plotting the expected distribution against observed results.

\section{Combined-phenotype analyses}

We used an adaptive sum of powered scores (aSPU) simulation-based method to perform a combinedphenotype analysis incorporating univariate results from seven RBC traits in sixteen analytic subgroups that were combined using inverse-variance-weighted meta-analysis. To evaluate evidence for shared genetic effects across all seven $\mathrm{RBC}$ traits, we combined meta-analyzed univariate results with aSPU to generate a combined-phenotype $p$ value for each SNP $[28,69]$. In comparison with other available methods, we chose aSPU because it exhibited low type 1 error rate in simulations; accommodated direction of effect; and was computationally scalable to the millions of SNPs measured using 1000 Genomes Phase 3 imputed data [70]. We implemented aSPU using Julia 1.0 to optimize efficiency (https://github.com/kaskarn/aspu_julia).

aSPU incorporated univariate summary z-scores, calculated for each SNP across all 7 traits, to yield a single p-value evaluating whether one or more of the traits were associated with a given SNP. Briefly, the procedure estimates $\Sigma$, the $7 \times 7$ correlation of null z-scores across univariate results and draws $10^{11}$ Monte-Carlo samples from the multivariate $N_{7}(0, \hat{\Sigma})$ distribution. For each SNP $j$, the results for all 7 traits $z_{j 1}, \ldots, z_{j 7}$ are used to form a sequence of sums of powered scores: $\operatorname{SPU}(\gamma)=z_{1}^{\gamma}+\ldots$ $+z_{7}^{\gamma}$, where $\gamma=0,1, \ldots, 8$, plus $\operatorname{SPU}(\infty)=\max \left|S_{7}\right|$. Each powered score is compared to the distribution of the $10^{11}$ powered scores calculated using simulated null values with the same $\gamma$ to calculate a Monte-Carlo p-value. An overall SNP $p$-value ( $p_{a S P U}$, possible range: $\left[1 /\left(1+10^{11}\right)\right.$, $1]$ ), is calculated by comparing the minimum $p$-value across the sequence of powered scores to the reference distribution of minimum $p$-values across the sequence of powered scores computed using the simulated null data. The adaptive aspect of the test lies in the potential for different $\gamma$ values to yield the maximal SPU across SNPs, maintaining power compared to a test with only a single possible alternative hypothesis.

\section{Sensitivity analyses}

Sensitivity analyses were performed for combined-trait results by self-reported race/ethnicity among analytic subgroups with greater than 1000 participants (i.e., restricted to African Americans, Hispanics/Latinos, and European Americans). Given the number of known ancestry-specific variants driving blood trait values, it was necessary to ensure that all self-reported race/ethnic groups be evaluated individually for associations that may be undetectable in the larger population. Meta- analyses of univariate summary statistics followed by combined-phenotype analysis were performed within each self-reported race/ethnicity using the same methods described above for the overall study population to identify genome-wide association signals $(p<5 \mathrm{E}-09)$.

We also examined whether there was evidence of significant trait-specific loci that were not identified in combined-phenotype analyses. Meta-analyses of each univariate $\mathrm{RBC}$ trait across all analytic subpopulations, as described above, were evaluated for association signals exceeding genome-wide significance $(p<5 \mathrm{E}-09)$. Although RBC traits are expected to share genetic underpinnings, particularly within pairs of correlated traits, association signals which were trait-specific in the wellpowered UK BioBank blood trait GWAS suggest that each trait has its own unique suite of associations [12].

Finally, in an attempt to examine the influence of the previously identified $3.7 \mathrm{~kb}$ structural variant esv3637548 in the $H B A 1 / 2$ region of chromosome 16 , we also adjusted for esv3637548 dosage $\left(r^{2}=0.86\right)$ in the MEGAgenotyped subgroup [28]. This structural variant either overlaps or has the potential to affect chromatic accessibility for multiple variants at this locus, but is present as both a duplication and a deletion. The duplication was not able to be imputed, and the deletion only met imputation quality criteria in the MEGA-genotyped study population, hence esv3637548 could not be evaluated within the entire study population in which this variant may be present. To evaluate the potential effect of this variant on each lead SNP reported as independent within our study, unadjusted combined-phenotype $p$ values were therefore compared to $\mathrm{p}$-values after conditioning on esv3637548.

\section{Generalization of previously reported associations to PAGE}

All SNPs located within $500 \mathrm{~kb}$ of a variant previously reported for any $\mathrm{RBC}$ trait were evaluated for evidence of association in the combined-phenotype analysis as well as each individual trait analysis. A generalization significance threshold of 1.07E-4 was calculated using Bonferroni correction for the previous number of onemegabase genomic regions for which one or more genome-wide-significant variants were reported for one or more RBC traits $(n=466$, representing 1308 index SNPs previously reported for one or more of the seven RBC traits we evaluated). We first reported trait-specific associations-i.e., index variants that have been reported by trait. We did not report loci containing a SNP that exceeded genome-wide-significance for the first time in one $\mathrm{RBC}$ trait but were previously reported for another trait as discovery associations; therefore, we also used the aforementioned significance threshold to evaluate 
generalization of association signals in each trait across all known loci.

\section{Identification of conditionally independent association signals}

Iterative conditional analysis was performed to identify all independent, genome-wide-significant combinedphenotype lead SNPs as described above. To avoid identifying SNPs as independent that were in long-range LD, we began by conditioning on the top SNP within ten megabase windows on each chromosome. To identify independent SNPs, linear models were extended to include all PAGE combined-phenotype lead SNPs on shared chromosomes using the same methods described above for univariate analysis, with an added covariate to include the dosage information for each participant at each lead SNP. Following each round of conditioning, aSPU was re-run on conditioned results. Additional rounds of conditional analyses were performed as an iterative process until no genome-wide-significant SNPs remained in the combined phenotype analysis.

\section{Publicly available expression quantitative trait locus (eQTL) analysis}

To help prioritize candidate causal gene-variant associations at identified loci, we evaluated all available lead SNPs within significant loci in relevant available tissues (whole blood, liver, spleen, and thyroid) for evidence of association with gene expression using the Genotype Tissue Expression (GTEx) portal [49].

\section{Supplementary information}

Supplementary information accompanies this paper at https://doi.org/10. 1186/s12864-020-6626-9.

Additional file 1: Figure S1. Manhattan and Quantile-Quantile plots for individual RBC traits in the total study population. In Manhattan plots, previously reported loci (published index SNP reported $p<5 \mathrm{E}-08$ within $500 \mathrm{~kb}$ of PAGE combined-phenotype lead SNP) are shown in purple; previously unreported loci with a PAGE lead SNP $p<5$ E-09 are shown in green. In Q-Q plots, all (black) $p$-values and $\mathrm{p}$-values for variants $>500 \mathrm{~kb}$ from a previously reported significant variant for any $R B C$ trait (blue) are both shown. Figure S2. Evidence of genetic associations shared across correlated RBC traits. X-axis: chromosome and position (top) and rsid (bottom) for each combined-phenotype lead SNP. Y-axis: trait-specific $\log _{10}(\mathrm{p}$-values), with increased intensity representing higher significance, for each combined-phenotype lead SNP. P-values scaled to a maximum $\log _{10}$ value of 25 for improved interpretation. Figure S3. Locus-Zoom plots of the association between rs6573766 and RBCC in PAGE African Americans on an African American LD background (A), Hispanics/Latinos on a Hispanic/Latino LD background (B), and European Americans on a European LD background (C). Each point represents one SNP; $x$-axis: increasing position on chromosome 14 left to right; $y$-axis: $-\log _{10}(p$-value) of the association with MCH SNP correlation with the lead SNP $\left(r^{2}\right)$ is colored according to the legend in Figure S3A. Annotated Refseq genes proximal to the lead SNP are shown by position above the $X$ axis. Figure S4. Locus-Zoom plot of the association between MCH (A) and MCV (B) and rs 145548796 in the total MEGA study population. Each point represents one SNP; $x$-axis: increasing position on chromosome 6 left to right; $y$-axis: - $\log _{10}(p$-value) of the association with MCH SNP correlation with the lead SNP $\left(r^{2}\right)$ is colored according to the legend in Figure S4A. Annotated Refseq genes proximal to the lead SNP are shown by position above the $X$ axis.

Additional file 2. Twelve supplemental tables supporting findings reported in the main text. Tables cover trait, genotyping, and QC description; ancestry- and trait-specific findings for combined-phenotype lead SNPs; sensitivity analysis of a deletion at the HBA1/2 locus; generalization of previously reported findings to PAGE study populations; and eQTL findings for PAGE lead SNPs in relevant tissue types.

\section{Abbreviations}

ARIC: Atherosclerosis Risk in Communities study; aSPU: adaptive sum of powered scores; CARDIA: Coronary Artery Risk Development in Young Adults Study; eQTL: expression quantitative trait locus; GTEx: Genotype Tissue Expression project; GWAS: Genome-wide association study; HCHS/

SOL: Hispanic Community Health Study/Study of Latinos; HCT: Hematocrit; HGB: Hemoglobin concentration; kb: kilobase; LD: Linkage disequilibrium; MCH: Mean corpuscular hemoglobin; MCHC: Mean corpuscular hemoglobin concentration; MCV: Mean corpuscular volume; MEGA: Multi-ethnic genotyping array; PAGE: Population Architecture using Genomics and Epidemiology study; RBC: Red blood cell; RBCC: Red blood cell count; RDW: Red cell distribution width; SNP: Simple nucleotide polymorphism; WHI: Women's Health Initiative study

\section{Acknowledgements}

The contents of this paper are solely the responsibility of the authors and do not necessarily represent the official views of the National Institutes of Health $(\mathrm{NIH})$. The PAGE consortium thanks the staff and participants of all PAGE studies for their contributions. We thank R. Williams and M. Ginoza for providing assistance with program coordination. The complete list of PAGE members can be found at http://www.pagestudy.org.

\section{Authors' contributions}

All contributing authors were given the opportunity to review and comment on the final manuscript and accompanying materials, and have approved submission for publication. Overall project supervision and management: $\mathrm{CJH}, \mathrm{CLA}, \mathrm{KEN}, \mathrm{APR}, \mathrm{MG}, \mathrm{RT}$. Genotyping and quality control: GLW, SB, MF, RJFL, CLK. Phenotype harmonization: CJH, SAB, WT, BT. Association and secondary analyses: CJH, ARB, CS, RT, MG. Manuscript preparation: CJH, ARB, $S A B, L M R, H M H, C S, M G, L A H, Y L, D L, A P R, K E N$.

\section{Authors' information}

N/A

\section{Funding}

None of the funding bodies described herein played a role in the design of the study; collection, analysis, and interpretation of data; or in writing the manuscript. The Population Architecture Using Genomics and Epidemiology (PAGE) program is funded by the National Human Genome Research Institute (NHGRI) with co-funding from the National Institute on Minority Health and Health Disparities (NIMHD). Assistance with data management, data integration, data dissemination, genotype imputation, ancestry deconvolution, population genetics, analysis pipelines and general study coordination was provided by the PAGE Coordinating Center (NIH U01HG007419). Genotyping services were provided by the Center for Inherited Disease Research (CIDR). The CIDR is fully funded through a federal contract from the NIH to The Johns Hopkins University, contract number HHSN268201200008l. Genotype data quality control and quality assurance services were provided by the Genetic Analysis Center in the Biostatistics Department of the University of Washington, through support provided by the CIDR contract. The data and materials included in this report result from collaboration between the following studies and organizations: ARIC, BioMe Biobank, CARDIA, HCHS/ SOL, PAGE Global Reference Panel and WHI. The BioMe Biobank received funding for the PAGE IPM BioMe Biobank study through the National Human Genome Research Institute (NIH U01HG007417). Primary funding support to $\mathrm{KEN}, \mathrm{RT}, \mathrm{HMH}, \mathrm{CLA}, \mathrm{CJH}, \mathrm{MF}$, and D-YL (as part of HCHS/SOL) is provided by U01HG007416. Additional support was provided via R01DK101855. The Atherosclerosis Risk in Communities Study (ARIC) was carried out as a collaborative study supported by R01HL087641, R01HL59367 and R01HL086694; National Human Genome Research Institute contract U01HG004402; and 
National Institutes of Health contract HHSN268200625226C. The Coronary Artery Risk Development in Young Adults Study (CARDIA) is supported by contracts HHSN268201300025C, HHSN268201300026C, HHSN268201300027C, HHSN268201300028C, HHSN268201300029C, and HHSN268200900041C from the National Heart, Lung, and Blood Institute (NHLBI), the Intramural Research Program of the National Institute on Aging (NIA), and an intra agency agreement between NIA and NHLBI (AG0005).

The HCHS/SOL study was carried out as a collaborative study supported by contracts from the National Heart, Lung and Blood Institute (NHLBI) to the University of North Carolina (N01-HC65233), University of Miami (N01HC65234), Albert Einstein College of Medicine (N01-HC65235), Northwestern University (N01-HC65236) and San Diego State University (N01-HC65237). The WHI program is funded by the NHLBI, NIH, US Department of Health and Human Services through contracts HHSN268201100046C, HHSN268201100001C, HHSN268201100002C, HHSN268201100003C, HHSN268201100004C and HHSN271201100004C. CJH, HMH, and LMR were also supported by NHLBI training grant HL129982. HMH was also supported by NHLBI training grant T32 HL007055 and ADA grant \#1-19-PDF-045. D-YL was also supported by R01CA082659, R01GM047845, and P01CA142538. CLA, ARB, CMS, HMH, and CJH were also supported by R01HL 142825.

\section{Availability of data and materials}

Complete summary level results are available through dbGaP (http://www. ncbi.nlm.nih.gov/projects/gap/cgi-bin/study.cgi?study_id=phs000356).

\section{Ethics approval and consent to participate}

All participating studies listed in the methods section obtained Institutional Review Board approval and written informed consent from all participants. Specifically, for the ARIC study IRBS at the following institutions approved the inclusion of this data in PAGE genomics studies: University of North Carolina at Chapel Hill, Johns Hopkins University, University of Minnesota, and University of Mississippi Medical Center. Use of HCHS/SOL study data was approved by IRBs from the following institutions: Northwestern University, Albert Einstein School of Medicine, University of Miami, and San Diego State University. Use of the BioMe Biobank data was approved by the Mt. Sinai IRB. Use of the WHI data was approved by the Fred Hutchinson Cancer Research Center IRB.

\section{Consent for publication}

N/A

\section{Competing interests}

All authors declare that they have no competing financial or non-financial interests.

\section{Author details \\ ${ }^{1}$ University of North Carolina Gillings School of Public Health, 135 Dauer Dr, Chapel Hill, NC 27599, USA. 'University of Virginia Center for Public Health Genomics, 1355 Lee St, Charlottesville, VA 22908, USA. ${ }^{3}$ Fred Hutchinson Cancer Research Center, 1100 Fairview Ave N, Seattle, WA 98109, USA. ${ }^{4}$ Department of Genetics, University of North Carolina at Chapel Hill, 120 Mason Farm Road, Chapel Hill, NC 27599, USA. ${ }^{5}$ University of Washington, 1730 Minor Ave, Ste 1360, Seattle, WA 98101, USA. 'Stanford University School of Medicine, 291 Campus Dr, Stanford, CA 94305, USA. ${ }^{7}$ Vanderbilt University, 2525 West End Ave \#1100, Nashville, TN 37203, USA. ${ }^{8}$ University of Minnesota, 420 Delaware St SE, Minneapolis, MN 55455, USA. ${ }^{9}$ Rutgers University, 683 Hoes Ln W, Piscataway, NJ 08854, USA. ${ }^{10}$ University of Texas Houston, 7000 Fannin Street, Houston, TX 77030, USA. ${ }^{11}$ National Human Genome Research Institute, 31 Center Dr, Bethesda, MD 20894, USA. \\ ${ }^{12}$ University of Washington, 1705 NE Pacific St, Seattle, WA 98195, USA. \\ ${ }^{13}$ Icahn School of Medicine at Mount Sinai, 1468 Madison Ave, New York, NY 10029, USA.}

Received: 24 October 2019 Accepted: 26 February 2020

Published online: 14 March 2020

\section{References}

1. Buttarello M. Laboratory diagnosis of anemia: are the old and new red cell parameters useful in classification and treatment, how? Int J Lab Hematol. 2016;38(Suppl 1):123-32.
2. Migone de Amicis M, Chivite D, Corbella X, Cappellini MD, Formiga F. Anemia is a mortality prognostic factor in patients initially hospitalized for acute heart failure. Intern Emerg Med. 2017 Sep;12(6):749-56.

3. Dai Y, Konishi H, Takagi A, Miyauchi K, Daida H. Red cell distribution width predicts short- and long-term outcomes of acute congestive heart failure more effectively than hemoglobin. Exp Ther Med. 2014;8(2):600-6.

4. Kellert L, Martin E, Sykora M, Bauer H, Gussmann P, Diedler J, et al. Cerebral oxygen transport failure?: decreasing hemoglobin and hematocrit levels after ischemic stroke predict poor outcome and mortality: STroke: RelevAnt impact of hemoGlobin, hematocrit and transfusion (STRAIGHT)--an observational study. Stroke. 2011 Oct;42(10):2832-7.

5. Dzierzak E, Philipsen S. Erythropoiesis: development and differentiation. Cold Spring Harb Perspect Med. 2013;3(4):a011601.

6. Barlas RS, Honney K, Loke YK, McCall SJ, Bettencourt-Silva JH, Clark AB, et al. Impact of hemoglobin levels and Anemia on mortality in acute stroke: analysis of UK regional registry data, systematic review, and meta-analysis. J Am Heart Assoc. 2016;17:5(8).

7. Whitfield JB, Martin NG. Genetic and environmental influences on the size and number of cells in the blood. Genet Epidemiol. 1985;2(2):133-44.

8. Wright FA, Sullivan PF, Brooks Al, Zou F, Sun W, Xia K, et al. Heritability and genomics of gene expression in peripheral blood. Nat Genet. 2014 May; 46(5):430-7.

9. Patel KV. Variability and heritability of hemoglobin concentration: an opportunity to improve understanding of anemia in older adults. Haematologica. 2008 Sep;93(9):1281-3.

10. Brendel C, Guda S, Renella R, Bauer DE, Canver MC, Kim Y-J, et al. Lineagespecific BCL11A knockdown circumvents toxicities and reverses sickle phenotype. J Clin Invest. 2016 Oct 3;126(10):3868-78.

11. Trakarnsanga K, Wilson MC, Lau W, Singleton BK, Parsons SF, Sakuntanaga P, et al. Induction of adult levels of $\beta$-globin in human erythroid cells that intrinsically express embryonic or fetal globin by transduction with KLF1 and BCL11A-XL. Haematologica. 2014 Nov;99(11):1677-85.

12. Astle WJ, Elding $H$, Jiang $T$, Allen $D$, Ruklisa $D$, Mann AL, et al. The allelic landscape of human blood cell trait variation and links to common complex disease. Cell. 2016;167(5):1415-1429.e19.

13. Ferreira MAR, Hottenga J-J, Warrington NM, Medland SE, Willemsen G, Lawrence RW, et al. Sequence variants in three loci influence monocyte counts and erythrocyte volume. Am J Hum Genet. 2009 Nov;85(5):745-9.

14. Yang Q, Kathiresan S, Lin J-P, Tofler GH, O'Donnell CJ. Genome-wide association and linkage analyses of hemostatic factors and hematological phenotypes in the Framingham Heart Study. BMC Med Genet. 2007;8(Suppl 1):S12.

15. Chambers JC, Zhang W, Li Y, Sehmi J, Wass MN, Zabaneh D, et al. Genomewide association study identifies variants in TMPRSS6 associated with hemoglobin levels. Nat Genet. 2009 Nov;41(11):1170-2.

16. Lo KS, Wilson JG, Lange LA, Folsom AR, Galarneau G, Ganesh SK, et al. Genetic association analysis highlights new loci that modulate hematological trait variation in Caucasians and African Americans. Hum Genet. 2011;129(3):307-17.

17. Yasukochi Y, Sakuma J, Takeuchi I, Kato K, Oguri M, Fujimaki T, et al. Identification of nine novel loci related to hematological traits in a Japanese population. Physiol Genomics. 2018 Sep 1;50(9):758-69.

18. Chen Z, Tang H, Qayyum R, Schick UM, Nalls MA, Handsaker R, et al. Genomewide association analysis of red blood cell traits in African Americans: the COGENT network. Hum Mol Genet. 2013 Jun 15;22(12):2529-38.

19. van Rooij FJA, Qayyum R, Smith AV, Zhou Y, Trompet S, Tanaka T, et al. Genome-wide trans-ethnic meta-analysis identifies seven genetic loci influencing erythrocyte traits and a role for RBPMS in erythropoiesis. Am J Hum Genet. 2017 Jan 5;100(1):51-63.

20. van der Harst P, Zhang W, Mateo Leach I, Rendon A, Verweij N, Sehmi J, et al. Seventy-five genetic loci influencing the human red blood cell. Nature. 2012 Dec 20;492(7429):369-75.

21. Soranzo N, Spector TD, Mangino M, Kühnel B, Rendon A, Teumer A, et al. A genome-wide meta-analysis identifies 22 loci associated with eight hematological parameters in the HaemGen consortium. Nat Genet. 2009 Nov;41(11):1182-90

22. Pistis G, Okonkwo SU, Traglia M, Sala C, Shin S-Y, Masciullo C, et al. Genome wide association analysis of a founder population identified TAF3 as a gene for MCHC in humans. PLoS One. 2013 Jul 31;8(7):e69206.

23. Okada Y, Kamatani Y. Common genetic factors for hematological traits in humans. J Hum Genet. 2012 Mar;57(3):161-9. 
24. Li J, Glessner JT, Zhang H, Hou C, Wei Z, Bradfield JP, et al. GWAS of blood cell traits identifies novel associated loci and epistatic interactions in Caucasian and African-American children. Hum Mol Genet. 2013 Apr 1;22(7): 1457-64.

25. Kullo IJ, Ding K, Jouni H, Smith CY, Chute CG. A genome-wide association study of red blood cell traits using the electronic medical record. PLoS One. 2010;28:5(9)

26. Kamatani Y, Matsuda K, Okada Y, Kubo M, Hosono N, Daigo Y, et al. Genome-wide association study of hematological and biochemical traits in a Japanese population. Nat Genet. 2010 Mar;42(3):210-5.

27. lotchkova V, Huang J, Morris JA, Jain D, Barbieri C, Walter K, et al. Discovery and refinement of genetic loci associated with cardiometabolic risk using dense imputation maps. Nat Genet. 2016 Sep 26;48(11):1303-12.

28. Hodonsky CJ, Jain D, Schick UM, Morrison JV, Brown L, McHugh CP, et al. Genome-wide association study of red blood cell traits in Hispanics/Latinos: the Hispanic community health study/study of Latinos. PLoS Genet. 2017 Apr 28;13(4):e1006760.

29. Ganesh SK, Zakai NA, van Rooij FJA, Soranzo N, Smith AV, Nalls MA, et al. Multiple loci influence erythrocyte phenotypes in the CHARGE Consortium. Nat Genet. 2009 Nov;41(11):1191-8.

30. Ding K, de Andrade M, Manolio TA, Crawford DC, Rasmussen-Torvik LJ, Ritchie MD, et al. Genetic variants that confer resistance to malaria are associated with red blood cell traits in African-Americans: an electronic medical record-based genome-wide association study. G3 (Bethesda). 2013; 3(7):1061-8.

31. Kanai M, Akiyama M, Takahashi A, Matoba N, Momozawa Y, Ikeda M, et al. Genetic analysis of quantitative traits in the Japanese population links cell types to complex human diseases. Nat Genet. 2018 Feb 5;50(3):390-400.

32. Bustamante CD, Burchard EG, De la Vega FM. Genomics for the world. Nature. 2011:475(7355):163-5.

33. DIAbetes Genetics Replication And Meta-analysis (DIAGRAM) Consortium, Asian Genetic Epidemiology Network Type 2 Diabetes (AGEN-T2D) Consortium, South Asian Type 2 Diabetes (SAT2D) Consortium, Mexican American Type 2 Diabetes (MAT2D) Consortium, Type 2 Diabetes Genetic Exploration by Nex-generation sequencing in muylti-Ethnic Samples (T2DGENES) Consortium, Mahajan A, et al. Genome-wide trans-ancestry metaanalysis provides insight into the genetic architecture of type 2 diabetes susceptibility. Nat Genet. 2014;46(3):234-44.

34. Kichaev G, Roytman M, Johnson R, Eskin E, Lindström S, Kraft P, et al. Improved methods for multi-trait fine mapping of pleiotropic risk loci. Bioinformatics. 2017 Jan 15;33(2):248-55.

35. Schick UM, Jain D, Hodonsky CJ, Morrison JV, Davis JP, Brown L, et al. Genomewide association study of platelet count identifies ancestry-specific loci in Hispanic/Latino Americans. Am J Hum Genet. 2016 Feb 4;98(2):229-42

36. Parra EJ, Mazurek A, Gignoux CR, Sockell A, Agostino M, Morris AP, et al. Admixture mapping in two Mexican samples identifies significant associations of locus ancestry with triglyceride levels in the BUD13/ZNF259/ APOA5 region and fine mapping points to rs964184 as the main driver of the association signal. PLoS One. 2017 Feb 28;12(2):e0172880.

37. 1000 Genomes Project Consortium, Auton A, Brooks LD, Durbin RM, Garrison EP, Kang HM, et al. A global reference for human genetic variation. Nature. 2015;526(7571):68-74.

38. Li YR, Keating BJ. Trans-ethnic genome-wide association studies: advantages and challenges of mapping in diverse populations. Genome Med. 2014 Oct 31;6(10):91.

39. Petrovski S, Goldstein DB. Unequal representation of genetic variation across ancestry groups creates healthcare inequality in the application of precision medicine. Genome Biol. 2016;17(1):157.

40. Bigdeli TB, Genovese G, Georgakopoulos P, et al. Contributions of common genetic variants to risk of schizophrenia among individuals of African and Latino ancestry. Mol Psychiatry. 2019. https://doi.org/10.1038/s41380-019-0517-y.

41. Girirajan S. Missing heritability and where to find it. Genome Biol. 2017 May 11;18(1):89.

42. Kim H, Grueneberg A, Vazquez Al, Hsu S, de Los Campos G. Will big data close the missing heritability gap? Genetics. 2017 Sep 11;207(3):1135-45

43. Pickrell JK, Berisa T, Liu JZ, Ségurel L, Tung JY, Hinds DA. Detection and interpretation of shared genetic influences on 42 human traits. Nat Genet. 2016 May 16:48(7):709-17.

44. Park H, Li X, Song YE, He KY, Zhu X. Multivariate analysis of anthropometric traits using summary statistics of genome-wide association studies from GIANT Consortium. PLoS One. 2016 Oct 4;11(10):e0163912.
45. Chami N, Chen M-H, Slater AJ, Eicher JD, Evangelou E, Tajuddin SM, et al. Exome genotyping identifies pleiotropic variants associated with red blood cell traits. Am J Hum Genet. 2016 Jul 7;99(1):8-21.

46. Matise TC, Ambite JL, Buyske S, Carlson CS, Cole SA, Crawford DC, et al. The next PAGE in understanding complex traits: design for the analysis of population architecture using genetics and epidemiology (PAGE) study. Am J Epidemiol. 2011 Oct 1;174(7):849-59.

47. Wei P, Cao Y, Zhang Y, Xu Z, Kwak I-Y, Boerwinkle E, et al. On robust association testing for quantitative traits and rare variants. G3 (Bethesda). 2016;6(12):3941-50.

48. Gerhard GS, Paynton BV, DiStefano JK. Identification of genes for hereditary hemochromatosis. Methods Mol Biol. 2018;1706:353-65.

49. GTEx Consortium. Human genomics. The genotype-tissue expression (GTEx) pilot analysis: multitissue gene regulation in humans. Science. 2015; 348(6235):648-60.

50. Lambert SA, Abraham G, Inouye M. Towards clinical utility of polygenic risk scores. Hum Mol Genet. 2019;28(R2):R133-42.

51. Martin AR, Kanai M, Kamatani Y, Okada Y, Neale BM, Daly MJ. Clinical use of current polygenic risk scores may exacerbate health disparities. Nat Genet. 2019 Mar 29;51(4):584-91.

52. Barbeira AN, Dickinson SP, Bonazzola R, Zheng J, Wheeler HE, Torres JM, et al. Exploring the phenotypic consequences of tissue specific gene expression variation inferred from GWAS summary statistics. Nat Commun. 2018 May 8;9(1):1825.

53. Peterson RE, Kuchenbaecker K, Walters RK, Chen C-Y, Popejoy AB, Periyasamy S, et al. Genome-wide association studies in ancestrally diverse populations: opportunities, methods, pitfalls, and recommendations. Cell. 2019 Oct 17;179(3):589-603.

54. Evans TC, Jehle D. The red blood cell distribution width. J Emerg Med. 1991; 9(Suppl 1):71-4

55. Monzon CM, Beaver BD, Dillon TD. Evaluation of erythrocyte disorders with mean corpuscular volume (MCV) and red cell distribution width (RDW). Clin Pediatr (Phila). 1987;26(12):632-8.

56. Wojcik GL, Graff M, Nishimura KK, Tao R, Haessler J, Gignoux CR, et al. Genetic analyses of diverse populations improves discovery for complex traits. Nature. 2019 Jun 19;570(7762):514-8.

57. Hsieh Y-P, Chang C-C, Kor C-T, Yang Y, Wen Y-K, Chiu P-F. The predictive role of red cell distribution width in mortality among chronic kidney disease patients. PLoS One. 2016 Dec 1;11(12):e0162025.

58. Tseliou E, Terrovitis JV, Kaldara EE, Ntalianis AS, Repasos E, Katsaros L, et al. Red blood cell distribution width is a significant prognostic marker in advanced heart failure, independent of hemoglobin levels. Hell J Cardiol. 2014 Dec:55(6):457-61.

59. Panwar B, Judd SE, Warnock DG, McClellan WM, Booth JN, Muntner $P$, et al. Hemoglobin concentration and risk of incident stroke in community-living adults. Stroke. 2016;47(8):2017-24.

60. Solovieff N, Cotsapas C, Lee PH, Purcell SM, Smoller JW. Pleiotropy in complex traits: challenges and strategies. Nat Rev Genet. 2013 Jul;14(7):483-95.

61. Chesmore K, Bartlett J, Williams SM. The ubiquity of pleiotropy in human disease. Hum Genet. 2018 Jan;137(1):39-44.

62. Gaggin HK, Dec GW. The role of treatment for Anemia as a therapeutic target in the Management of Chronic Heart Failure: insights after RED-HF. Curr Treat Options Cardiovasc Med. 2014;16(1):279.

63. Morales J, Welter D, Bowler EH, Cerezo M, Harris LW, McMahon AC, et al. A standardized framework for representation of ancestry data in genomics studies, with application to the NHGRI-EBI GWAS catalog. Genome Biol. 2018;19(1):21.

64. Bien SA, Wojcik GL, Zubair N, Gignoux CR, Martin AR, Kocarnik JM, et al. Strategies for enriching variant coverage in candidate disease loci on a multiethnic genotyping array. PLoS One. 2016 Dec 14;11(12):e0167758.

65. MacArthur J, Bowler E, Cerezo M, Gil L, Hall P, Hastings E, et al. The new NHGRI-EBI catalog of published genome-wide association studies (GWAS catalog). Nucleic Acids Res. 2017 Jan 4;45(D1):D896-901.

66. Zheng X, Levine D, Shen J, Gogarten SM, Laurie C, Weir BS. A highperformance computing toolset for relatedness and principal component analysis of SNP data. Bioinformatics. 2012;28(24):3326-8.

67. Lin D-Y, Tao R, Kalsbeek WD, Zeng D, Gonzalez F, Fernández-Rhodes L, et al. Genetic association analysis under complex survey sampling: the Hispanic community health study/study of Latinos. Am J Hum Genet. 2014;95(6):675-88.

68. Willer CJ, Li Y, Abecasis GR. METAL: fast and efficient meta-analysis of genomewide association scans. Bioinformatics. 2010 Sep 1;26(17):2190-1. 
69. Kim J, Bai Y, Pan W. An adaptive association test for multiple phenotypes with GWAS summary statistics. Genet Epidemiol. 2015 Dec;39(8):651-63.

70. Sitlani CM, Baldassari AR, Highland HM, Hodonsky CJ, Avery CL. Comparison of multiple phenotype association tests using summary statistics in genome-wide association studies. Wiley Online Library; 2019.

\section{Publisher's Note}

Springer Nature remains neutral with regard to jurisdictional claims in published maps and institutional affiliations.

Ready to submit your research? Choose BMC and benefit from:

- fast, convenient online submission

- thorough peer review by experienced researchers in your field

- rapid publication on acceptance

- support for research data, including large and complex data types

- gold Open Access which fosters wider collaboration and increased citations

- maximum visibility for your research: over $100 \mathrm{M}$ website views per year

At $\mathrm{BMC}$, research is always in progress.

Learn more biomedcentral.com/submissions 